\title{
Analysis of Antimicrobial Efficacy of Triphala and its Effectiveness in Decontaminating the Guttapercha Cones
}

\begin{abstract}
Manu Rana, ${ }^{1}$ Shiksha Kumari, ${ }^{2}$ Manoj Kumar Upadhyay ${ }^{3}$
${ }^{1}$ Department of Conservative Dentistry and Endodontics, College Of Medical Sciences, Bharatpur, Nepal, ${ }^{2}$ Department of Ayurveda (BalRoga), Saraswati Ayurved Hospital and Medical college, Mohali, Punjab, India, ${ }^{3}$ Department of Conservative Dentistry and Endodontics , M.B. Kedia Dental College Birgunj, Parsa, Nepal.

\section{ABSTRACT}

Background: The prime target of endodontic treatment is to maintain an aseptic conditions starting right from the access opening to the obturation of root canals. Many chemicals such as, hydrogen peroxide, chlorhexidine, etc. have been tried for GP decontamination but none of these compounds have been proven as fully effective. The purpose of this study was to evaluate the effectiveness of an ayurvedic alternative, Triphala for rapid decontamination of guttapercha cones. Materials and Methods: A concentrated extract of triphala was used to check for the antimicrobial efficacy using the agar well diffusion method. Presence of zones' of diffusion was identified against three common GP contaminants namely, E. coli, E. faecalis and S. aureus. New GP Cones, freshly taken out of the packet were then decontaminated for 1minute using triphalaand then placed in thioglycolate broth to check for the presence of turbidity. Results: The zones of inhibition on the agar plate were measured as $23 \mathrm{~mm}, 21 \mathrm{~mm}$ and $24 \mathrm{~mm}$ respectively. The broth remained clear even after 48 hours of incubation Conclusions: We conclude that triphala is indeed effective as a GP decontaminant and it holds promising future as a medium for storage of GP cones.
\end{abstract}

Keywords: decontamination; guttapercha cones; Triphala.

\section{INTRODUCTION}

A long time before modern medicine provided scientific evidence for the mind-body connection, the sages of India developed Ayurveda, which continues to be one of the world's most sophisticated and powerful mind-body health systems. More than a mere system of treating illness, Ayurveda is a science of life (Ayur $=$ life, Veda $=$ science or Knowledge). It offers a body of wisdom designed to help people stay vibrant and healthy while realizing their full human potential.

Triphala [three (tri) fruits (phala)] is a plantderived composition developed in India. The powder is a combination of three dried plants naming Terminalia bellerica, Terminalia chebula and Phyllanthus emblica with tannic acid being its principal constituent. ${ }^{1-3}$ Ithas been used in Indian traditional medicine for treatment of headaches, constipation and hepatic disorders. ${ }^{2-6}$ Initial studies have shown bacteriostatic or bactericidal effect of tannic acid on gram-positive and gram-negative pathogens. ${ }^{6}$ Compared to commonly used root canal irrigants, it is safe and is composed of compounds with proper physiologic effects in addition to its anti-oxidative and anti-inflammatory properties. ${ }^{4}$ The most important advantages of Triphala include easy access, low cost, long-term substantivity, less toxicity and absence of microbial resistance. ${ }^{6}$

The elementary target of endodontic therapy is to maintain an aseptic condition starting right from the access opening to the obturation of root canals. Eliminating or decreasing the number of microorganisms is of considerable importance for endodontic success. Guttapercha (GP) is an extract of plants of palaquiam of the Blanco genus of sapotaceae family, and was introduced to dentistry in 1847 by Edwin Truman. ${ }^{7,8}$ It still continues to maintain its position as an important dental material and has emerged as the prime root canal filling material. GP supplied commercially is not usually sterilized or decontaminated before obturation. Also, it cannot be sterilized by moist or dry heat as this carries a risk of physical deformation. However, chair side decontamination prior to obturation cannot be ignored.

Correspondence: Dr. Manu Rana, Department of Conservative Dentistry and Endodontics, College of Medical Sciences, Bharatpur, Nepal. Email: ranamanu86@gmail.com. Phone: +9779852030496. DOI: 10.3126/ jcmsn.v14i4.20062. Article received: 2018-05-30. Article accepted: 2018-11-10. 
Many chemicals such as, hydrogen peroxide, chlorhexidine, ethyl alcohol, polyvinyl pyrolidone iodine, quartenary ammonium compounds have been tried for GP decontamination. However, none of these methods have been proven as fully effective. The recommended method for decontamination of GP points consists of treating the cones using a $1 \%$ Sodium hypochlorite for 1 minute (Milton's solution), or $0.5 \%$ Sodium hypochlorite for 5 minutes (Dakin's solution). ${ }^{9}$ Here, the risk of Sodium hypochlorite causing crystal deposition within the canals which can impede the obturation cannot be ignored. The purpose of this study was to evaluate the effectiveness of an ayurvedic alternative, Triphala for rapid decontamination of guttapercha cones.

\section{MATERIALS AND METHODS \\ Test organisms}

Reference strains of three most common GP contaminants, Eschericia coli, Enterococcus faecalis and Staphylococcus aureus were obtained from the IMTECH, Chandigarh ,India.

\section{Preparation of solution}

Triphala powder (Dabur, India) was dissolved in 10\% Dimethyl sulfoxide (DMSO) solution (Nice company) to prepare an irrigation solution at a concentration of $6 \mathrm{mg} / \mathrm{ml}$ by shaking beaker and keeping it on hot plate so that particles of powder were dissolved easily and the solution was filtered. $5.25 \%$ concentration of $\mathrm{NaOCl}$ solution (Maarc, shiva products) without preservatives was used in this study.

\section{Antimicrobial activity of Triphala}

The antibacterial activity of the extract was tested using Agar well diffusion technique. The reference strains were cultured overnight in thioglycolate broth, and the culture was streaked on a plate of blood agar. Three wells of $5 \mathrm{~mm} \times 5 \mathrm{~mm}$ measure were made with the help of a template on the surface of the agar plate. About $0.1 \mathrm{ml}$ of the extract was delivered into the well using a micropipette. The other two wells were filled with $5.25 \%$ of sodium hypochlorite and $0.9 \%$ normal saline as positive and negative controls, respectively. They were then incubated at $37{ }^{0} \mathrm{C}$ for 24 hours, and closely monitored for the development of clear zones around the extracts. The antibacterial activity was assessed by the diameter of the inhibition zone. A clear zone of inhibition was obtained against all the three organisms.

\section{Guttapercha decontamination}

A new pack of protaper universal guttapercha points F2 (Dentsply Tulsa dental specialities) were used for the procedure. The pack was opened under sterile conditions and four pointswere taken out using a sterile tweezer. The points were then placed inside the freshly prepared thioglycolate broth, and incubated for 24hours. Simultaneously, four new GP cones were removed and decontaminated for one minute in $90 \%$ triphala extract. The cones were then removed from the gel, and cleaned free of the extract using sterile gauze, and then incubated in thioglycolate broth for 24 hours. Both the tubes were then closely monitored for the development of turbidity.

\section{RESULTS}

The antimicrobial efficacy was assessed by the presence of zones of inhibition. Escherichia coli, Enterococcus faecalis and Staphylococcus aureus showed $24 \mathrm{~mm}, 21 \mathrm{~mm}$ and $24 \mathrm{~mm}$ inhibition zones respectively, which was almost equivalent to $5.25 \%$ Sodium hypochlorite used as the control as showed in Table 1. The decontaminating

\begin{tabular}{|llll|}
\hline \multicolumn{4}{|c|}{ Table 1. Zones of inhibition in millimetres (mm) } \\
obtained against the test organisms.
\end{tabular}

efficacy was then assessed by the occurrence of turbidity in the thioglycolate broth. The GP cones which were not decontaminated and directly placed in the broth developed turbidity. The cones decontaminated with triphala extract and then placed in the broth remained clear even after 24 hours, indicating the absence of the microbial contaminants.

\section{DISCUSSION}

Microorganisms and their by-products are considered the main cause of pulpal and periapical pathosis. ${ }^{10}$ The ingress of irritants from an infected root canal system through tubules, lateral or accessory canals, furcation canals, and the apical foramina are very well known to directly affect the surrounding apparatus. It is because the diameter of dentinal tubules is large enough to allow bacterial infiltration. The number of microorganisms within an infected root canal system may vary anywhere from 102 to more than 
108 as said by Sjogren. ${ }^{11}$ One study acclaimed that the microbes are present in all parts of the root canal system, including fins, and anastomoses and may be found at varying depths of up to $300 \mu \mathrm{m}$ within the dentinal tubules, from the pulpal end. ${ }^{12}$

The importance of GP decontamination to prevent any bacterial contamination of the root canal during the obturation procedure is now widely recognized in endodontic practice. Thus, it is imperative to employ a rapid, reliable, inexpensive and effective decontaminant. The results of the present study are similar with the study of Pujar et al, who compared the antimicrobial efficacy of $\mathrm{NaOCl}$, Triphala and Green tea polyphenols and found Triphala and Green tea to be effective against E.faecalis. $10 \%$ dimethyl sulfoxide (DMSO) was used as a solvent for Triphala. DMSO is a clean, safe, highly polar solvent that helps in bringing out the pure properties of all the components of the herb being dissolved. ${ }^{4}$

Sodium hypochlorite has a strong antibacterial and sporicidal effect, and acts by a mechanism involving the liberation of active chlorine, (a powerful oxidizing agent) which in turn inactivates the bacterial enzymes. Sodium hypochlorite 5.25\% has been found to be effective in decontaminating GP cones. However, it is imperative that after disinfection, the GP cone should be rinsed in ethyl alcohol to remove crystallized sodium hypochlorite before obturation as the crystals may impair the hermetic seal.

Plants have been used for ages in the treatment of medical illness. Throughout the history of mankind, many infectious diseases have been treated with herbs. Herbal medicines are now in great demand in the developing world for primary health care not because they are inexpensive but also for better cultural acceptability, better compatibility with the human body and minimal side effects. Triphala controls plaque from baseline and its activity is comparable to commonly available mouthwash chlorhexidine. ${ }^{13}$ Major advantage is that triphala extract has been found to be effective in decontaminating GP cones within one minute. To substantiate these results, further in depth studies incorporating more isolates from clinical samples are required.

\section{CONCLUSIONS}

Within the limits of this study, it can be concluded that triphala can be used effectively for decontaminating GP cones within a short duration, and holds a promising futureas a medium for storage of GP points.

\section{REFERENCES}

1. Prabhakar J, Senthilkumar M, Priya MS, Mahalakshmi K, Sehgal PK, Sukumaran VG. Evaluation of antimicrobial efficacy of herbal alternatives (Triphala and green tea polyphenols), MTAD, and 5\% sodium hypochlorite against Enterococcus faecalis biofilm formed on tooth substrate: an in vitro study. J Endod. 2010; 36(1):83-6.

2. Gomes BP, Vianna ME, Matsumoto CU, de Paula e Silva Rossi V, Zaia AA, Ferraz CCR, de Souza Filho FJ. Disinfection of gutta-percha cones with chlorhexidine and sodium hypochlorite. Oral Surg Oral Med Oral Pathol Oral RadiolEndod. 2005;100(4):512-7.

3. Park JB, Park NH. Effect of chlorhexidine on the in vitro and in vivo herpes simplex virus infection. Oral Surg Oral Med Oral Pathol. 1989;67(2):149-53.

4. Pujar M, Patil C, Kadam A. Comparison of antimicrobial efficacy of Triphala, (GTP) Green tea polyphenols and $3 \%$ of Sodium hypochlorite on Enterococcus faecalis biofilms formed on tooth substrate: in vitro. J Int Oral
Health; 2011: 3(2): 23-28.

5. Jagadish L, Kumar VK, Kaviyarasan V. Effect of Triphala on dental bio-film. Indian J Sci Technol. 2009;2(1):30-3.

6. Vani T, Rajani M, Sarkar S, Shishoo CJ. Antioxidant properties of the ayurvedic formulation triphala and its constituents. Pharmaceutical biology. 1997; 35(5):313-7.

7. Cruse WP, Bellizi R. A historic review of endodontics 1689-1963, Part I. J Endod 1980;6:495-9.

8. Jyoti BB. Phytotherapeutics in conservative dentistry and endodontics - a review. J Conserv Dent 2005;8:31-9.

9. Cardoso CL, Kotaka CR, Redmerski R, Guilhermetti M, Queiroz AF. Rapid decontamination of guttapercha cones with sodium hypochlorite. J Endod 2009; 25:498-501.

10. Ercan E, Ozekinci T, Atakul F, Gul K. Antibacterial Activity of $2 \%$ ChlorhexidineGluconate and 5.25\% Sodium hypochlorite in infected root canal: In Vivo Study. J Endod.2004; 30(2): 84-87.

11. SjogrenU, Figdor D, Sundqvist G. The 
antimicrobial effect of calcium hydroxide as a short term intracanal dressing. IntEndod $\mathrm{J}$, 1991; 24:119-125.

12. Horiba N, Maekawa Y, Matsumoto T, Nakamura H. (1990). A study of distribution of endotoxin in the dentinal wall of infected root canals. J Endod, 1990; 16: 331-334.

13. Srinagesha J, Pushpanjali K. Assessment of Antibacterial Efficacy of Triphala Against Mutans Streptococci-A Randomised Control Trial. Oral Health Prev Dent; 2011: 9(4): 387393.

Citation: Rana M, Kumari S, Upadhyay MK. Analysis of Antimicrobial Efficacy of Triphala and its effectiveness in Decontaminating the Guttapercha Cones. JCMS Nepal. 2018;14(4):217-20. 